\title{
Light curves and periods of Mira variables ${ }^{\star, \star \star, \star \star \star, \dagger}$
}

\author{
C. Alard $^{1,2}$, A. Terzan $^{3}$ and J. Guibert ${ }^{1,2}$ \\ 1 Centre d'Analyse des Images CNRS/INSU/MAMA, Observatoire de Paris, France \\ 2 Observatoire de Paris (DEMIRM)/URA 336 du CNRS, 77, Avenue Denfert-Rochereau F-75014 Paris, France \\ ${ }^{3}$ Observatoire de Lyon (CRAL), F-69561 Saint Genis Laval Cedex, France
}

Received April 7, 1995; accepted April 9, 1996

\begin{abstract}
Good distance indicators are needed in studies of the galactic structure. Pulsating variables can be used, but the problem is to find a homogenous sample obeying a period-luminosity relation with low scatter. In this paper, we show how such a sample can be produced using the Terzan catalogue of Variables and available Schmidt plates. We have selected 150 large amplitude variables discovered by Terzan in a field of 25 sq degrees near the Galactic Centre. A set of 22 red plates was scanned with the MAMA machine, providing time series for all the variables. The times series were analysed using both the periodogram and Renson's method. Periods could be derived for 122 stars of the sample showing clearly that most of these objects are Miras. As a conclusion we show that with some infrared photometry, these Miras could be used as good distance indicators in this region.
\end{abstract}

Key words: stars: AGB - infrared: stars - Galaxy: centre

\section{Introduction}

\subsection{AGB stars as probes of the galactic structure}

As demonstrated by Weinberg (1992), AGB stars can be very useful for the study of galactic structure. Weinberg used the variable stars of the IRAS survey (1988), and making the assumption that most of these objects are AGB stars having the same absolute magnitude, he could derive their spatial distribution. After correction for distance incompleteness, the IRAS variables traced a structure where the bulge was found as being elongated, and the beginning of two spiral arms was visible. This demonstrates the power of the method. However, the absolute $\mathrm{K}$ or bolometric magnitude of galactic AGB stars depends on the period (see, e.g., Whitelock et al. 1991; Glass et al. 1995, and references therein). For Miras, with such a $\mathrm{P}-\mathrm{L}$ relation, Weinberg's hypothesis according to which all Miras have the same absolute magnitude may produce a scatter of about 0.5 mag.

\footnotetext{
Send offprint requests to: C. Alard

${ }^{\star}$ Plate scanning done with MAMA. MAMA is developed and operated by INSU (Institut National des Sciences de l'Univers) ${ }^{\star \star}$ Based on observations made at the European Southern Observatory, La Silla, Chile

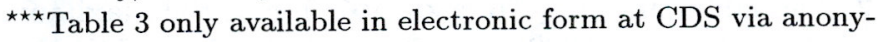
mous ftp cdsarc.u-strasbg.fr or 130.79.128.5

${ }^{\dagger}$ Figure 5 only available in electronic form at http://ww.edphys.fr
}

Taking into account the aforementioned periodluminosity relations, it might be possible to reduce this scatter, which will allow a better analysis of the galactic structure. goal of this study is to produce, from a set of available Schmidt plates, a sample of periodic AGB variables (Miras), with periods suitable to the derivation of the absolute luminosity and hence the distance of each object. However, both $\mathrm{K}$ and bolometric magnitudes involve infrared photometry, so our work should be further completed with observations in this spectral region.

\subsection{Miras}

Mira stars are large amplitude variables (more than $2 \mathrm{mag}$ in the visible for most of them) with long periods (from 150 to 500 days). In the HR diagram they are located on the AGB branch, a region occupied by old giant stars. These represent a late stage in the evolution of intermediate mass objects, connected with high mass loss rate leading to $\mathrm{PN}$ formation. Due to the intrinsic colours of these objects, red plates are very well suited for these variables. A long time basis covering a sufficient number of periods is also needed for period finding.

Our collection includes a set of 22 ESO red plates taken between 1980 and 1992. They cover the central part of the $10 \times 10$ degrees field in which one of us (A. T.), using the blink microscope technique, had discovered several thousands of variables (Terzan \& Gosset 1991 and references therein). Unfortunately, the time sampling is quite 
uneven. Consequently, irregular or semi-regular variables, for which it is impossible to fold all the measurements on a single cycle, are excluded from the present study.

\section{Data reduction}

\subsection{Scanning of the plates, and photometric calibration}

The MAMA machine was used in the mode in which it produces a digitised image of a small field centered on each of Terzan's stars. The magnitude determination was performed for the 22 red plates using a multi-threshold technique. Each plate was first calibrated by means of 55 standards (Terzan et al. 1982). For this purpose, a polynomial fit between photoelectric magnitudes and integrated photographic densities was made, for each threshold, with a least squares technique. Then, at each threshold where a given variable was detected and found as separated from possible neighbours, a magnitude was derived from the polynomial calibration. Finally, for each object and for each plate, the magnitudes obtained at the different thresholds were subsequently averaged.

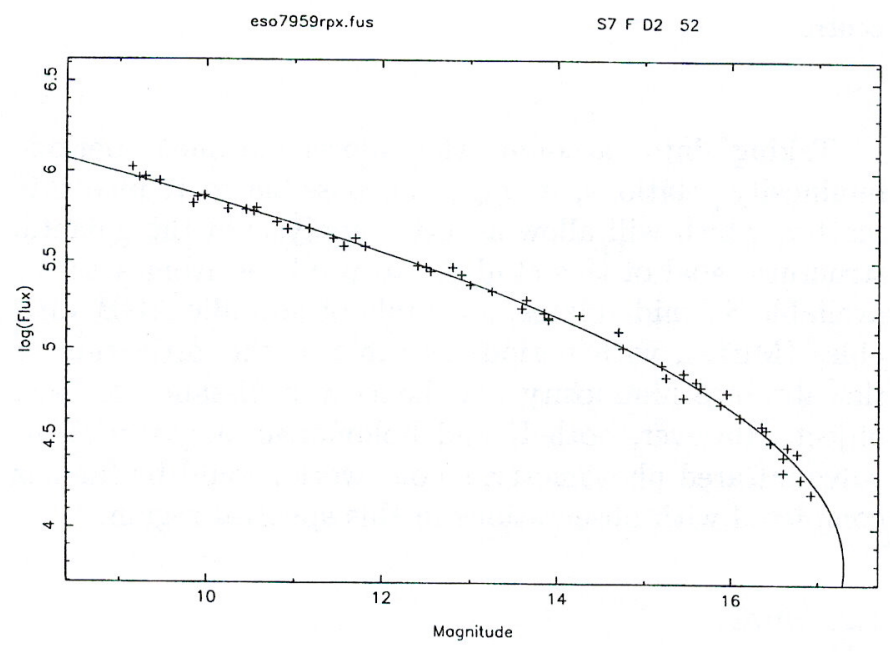

Fig. 1. Example of photometric calibration. "Flux" stands for the integrated photographic density

Table 1. Estimation of photometric errors

\begin{tabular}{ll}
\hline$R$ magnitude & magnitude error \\
\hline $10-12$ & 0.15 \\
$12-14$ & 0.10 \\
$14-16$ & 0.15 \\
$16-18$ & 0.20 \\
\hline
\end{tabular}

An estimate of the photometric accuracy can be derived from the residuals of the sequence fitting (Fig. 1) and the differences between the magnitudes obtained at the various thresholds. This estimate is given in Table 1 as a function of the $R$ magnitude.
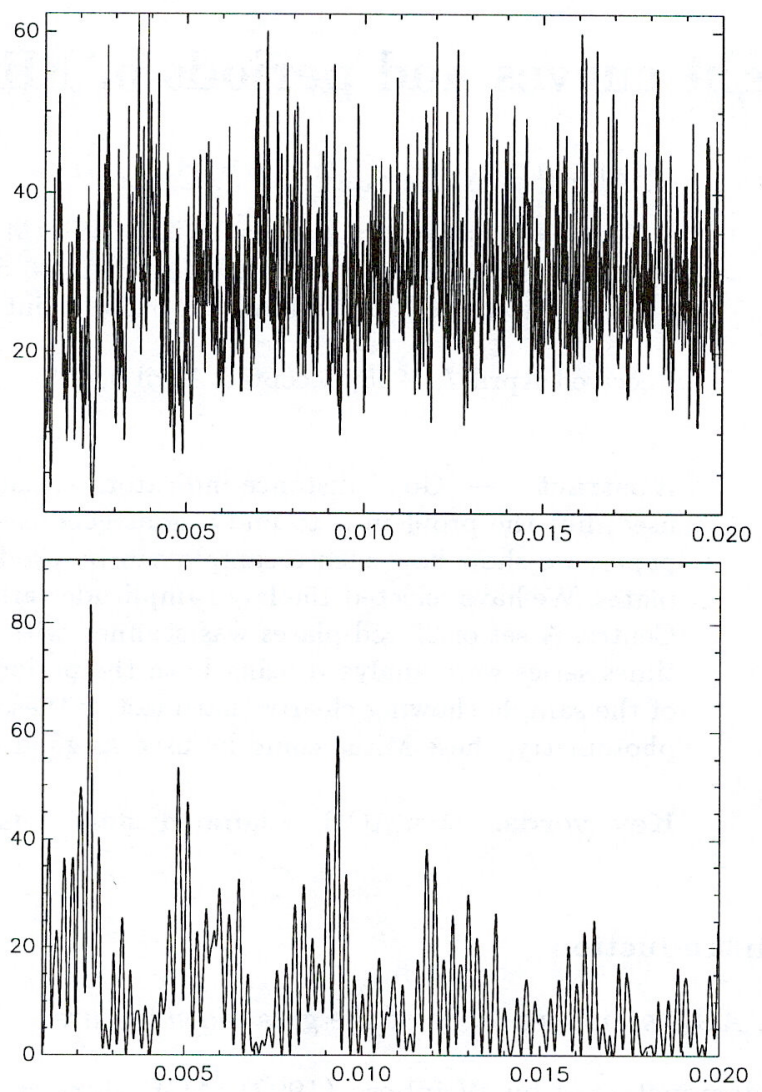

Fig. 2. Example of period determination. Top: Renson's method $\left(\theta_{1}\right)$; bottom: periodogram $\left(P_{\mathrm{X}}(\omega)\right)$

\subsection{Period determination}

The limited number of points as well as their very uneven time spacing, sometimes results in poor sampling of the light curve. In such a case, comparative studies demonstrate that Renson's (1978) method generally works better, but gives no estimate of the probability that the tested signal is really periodic. To estimate this probability it is well known that a Fourier method, like the periodogram (Deeming 1975; Scargle 1982) is optimal (Swingler 1989). So we preferred to use both Renson's and the periodogram methods to analyse the data.

In Figs. 2 and 3, we give an example of a time series analysis. The frequency spectra estimated with Renson's and the Periodogram methods are plotted in Fig. 2. The minimum of $\theta_{1}$ (Renson 1978), and the maximum of $P_{\mathrm{X}}(\omega)$ (Scargle 1982), are obtained at values of the frequency very close to $0.002366 \mathrm{day}^{-1}$, leading to a period of $422.6 \pm 2$ days. The derived phase diagram can be seen in Fig. 3. 
Table 2. Miras found in the IRAS Point Source Catalog (PSC)

\begin{tabular}{lcccccccccc}
\hline Terzan & PSC & period(d) & $\Delta \boldsymbol{\alpha}^{\prime \prime}$ & $\epsilon \boldsymbol{\alpha}^{\prime \prime}$ & $\Delta \delta^{\prime \prime}$ & $\epsilon \delta^{\prime \prime}$ & $12 \mu$ & $25 \mu$ & $\log \left(S_{12} / S_{25}\right)$ & $I_{\mathrm{V}}$ \\
\hline Ter0051 & $17159-2954$ & 298.7 & -10.4 & 44 & -0.5 & 6 & 1.020 & 0.717 & 0.153 & 0 \\
Ter0060 & $17163-2917$ & 307.3 & -10.4 & 38 & -1.9 & 6 & 2.992 & 1.531 & 0.291 & 9 \\
Ter0070 & $17168-2856$ & 523.6 & -03.9 & 28 & -0.3 & 6 & 15.27 & 6.673 & 0.360 & 9 \\
Ter0098 & $17183-2838$ & 286.4 & -03.9 & 30 & 0.2 & 5 & 2.480 & 1.303 & 0.280 & 8 \\
Ter0105 & $17186-2914$ & 422.7 & 03.9 & 25 & -1.6 & 5 & 14.07 & 5.470 & 0.423 & 9 \\
Ter0146 & $17201-2859$ & 387.9 & -05.2 & 28 & 0.2 & 5 & 3.739 & 1.857 & 0.304 & 9 \\
Ter0244 & $17241-2921$ & 435.9 & 05.2 & 41 & -1.1 & 7 & 2.529 & 1.467 & 0.237 & 9 \\
Ter0249 & $17242-3045$ & 404.5 & -03.9 & 31 & 4.4 & 5 & 6.929 & 5.037 & 0.138 & 1 \\
Ter0411 & $17268-3141$ & 517.6 & 10.4 & 41 & 6.6 & 8 & 8.789 & 4.836 & 0.259 & 9 \\
Ter0574 & $17303-2955$ & 305.1 & 09.1 & 39 & 1.4 & 6 & 4.130 & 2.709 & 0.183 & 9 \\
Ter0603 & $17321-3053$ & 438.6 & 03.9 & 48 & 1.8 & 9 & 10.69 & 5.725 & 0.271 & 8 \\
Ter0605 & $17322-3111$ & 222.5 & 19.5 & 35 & 0.9 & 8 & 2.586 & 1.878 & 0.139 & \\
\hline
\end{tabular}

Ter $=$ Terzan Number (Terzan et al. 1982); $\Delta \alpha=\alpha($ IRAS $)-\alpha($ Terzan $) ; \Delta \delta=\delta($ IRAS $)-\delta($ Terzan) $\epsilon \alpha$ and $\epsilon \delta$ are the dimensions of the IRAS error box (95\% confidence)

$I_{\mathrm{V}}$ (Variability Index) is the probability that $12 \mu$ and $25 \mu$ fluxes showed correlated variations $\left(\epsilon \alpha, \epsilon \delta, 12 \mu, 25 \mu\right.$ and $I_{\mathrm{V}}$ are taken from the IRAS PSC)

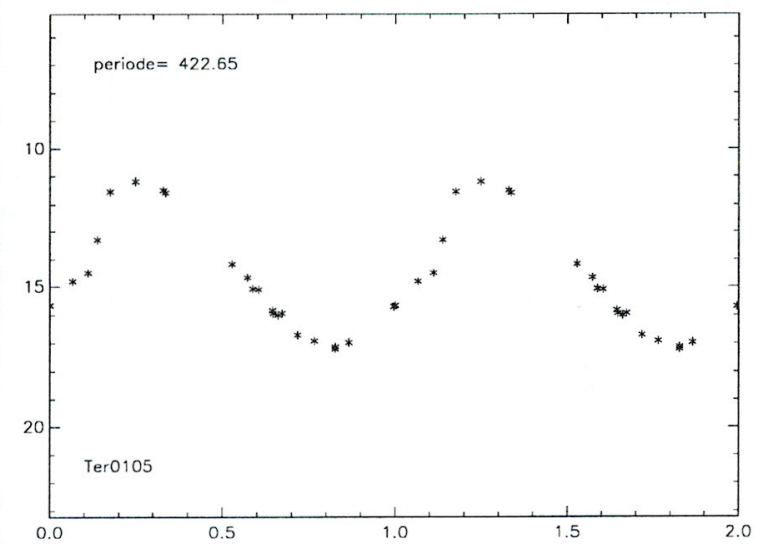

Fig. 3. Phase diagram using the period determinations shown in Fig. 2

The 150 times series were processed (Alard et al. 1993; Alard 1994), providing estimates of the period for each object, and the false alarm probability (Scargle 1982). Only the stars having a false alarm probability less than 5 percent, and a good agreement between the period estimates by Renson's and the Periodogram methods, were selected. A total of 122 objects satisfied these two conditions. The data are presented in Table 3 . The phase diagrams are reproduced in Fig. 5. The common origin of the abscissae corresponds to the Julian date J.D. 2443966.58 (Astronomical Almanach, M6)

\section{Comparison with the IRAS point source catalog}

We have found 122 stars with quite regular and repetitive variations, which are therefore considered as being Miras. Usually these objects have circumstellar shells emitting in the far infrared. A total of 12 stars were found to lie in

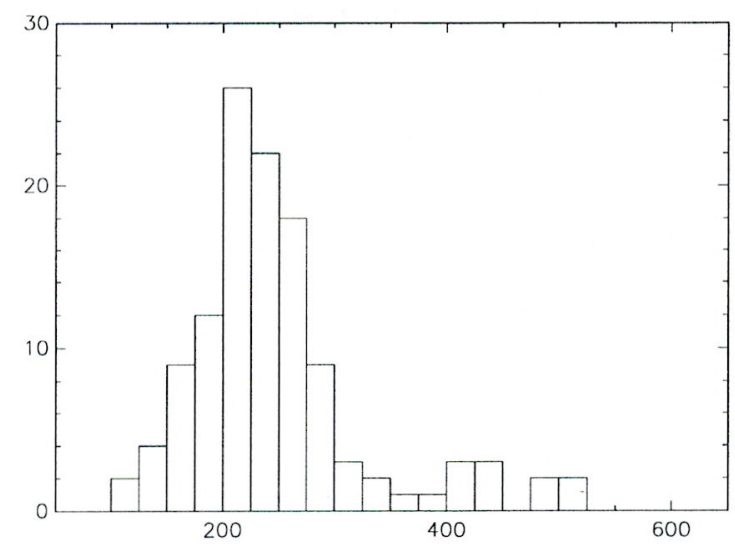

Fig. 4. Period Distribution of the Miras of our sample (periods are expressed in days)

the error box of an IRAS source. In Table 2, the ratio $\log \left(S_{12} / S_{25}\right)$, comprised between 0.1 and 0.5 , agrees well with the expected values for Miras (see, e.g., Vardya et al. 1986). Moreover, 10 objects, over a total number of 12 , have a variability probability exceeding 0.8 in the PSC. The number of IRAS observations for the two remaining LPVs (Ter0051 and Ter0605) was probably insufficient to suggest infrared variability. It could be rather surprising to find less than $10 \%$ of our (122) Miras in the IRAS PSC. However, there are two major reasons for the incompletness of the IRAS catalog in this region: the crowding on the one part, and the limited sensitivity of the survey on the other part.

\section{Conclusions}

Figure 4 shows that most of our stars are in the period range 150 to 300 days. The period-luminosity relations 
derived from infrared photometry (Whitelock et al. 1991; Glass et al. 1995), are quite accurate for this range of periods (sigma $\approx 0.2 \mathrm{mag}$ ). Using the period as derived above from the optical measurements, the absolute magnitude $K_{0}$ can be obtained. On the other hand, the mean apparent $J$ and $K$ magnitudes can be derived from infrared measurements. The extinction in this field is high in the visible, and probably also in the infrared. But the relation between the period and the color index $(J-K)_{0}$ (Glass et al. 1995), will give us the dereddened color, allowing us to derive both the extinction and distance modulus.

With this sample of stars, we demonstrate the interest of studying LPVs. This work will be continued for the other Terzan fields and, with the addition of some new plates, could be extended to variables of all types.

Acknowledgements. We are grateful to the MAMA team for assistance during the scans, to L. Pagani for providing the multithreshold algorithm, and to M. Feast for fruitful discussions.
We also thank the referee (Dr. C. Sterken) for valuable comments and suggestions.

\section{References}

Alard C., 1994, Ap\&SS 217, 155

Alard C., Terzan A., Guibert J., 1993, The Messenger 73, 31

Deeming T.J., 1975, Ap\&SS 36, 137

Glass I.S., Whitelock P.A., Catchpole R.M., Feast M.W., 1995, MNRAS 273, 383

Infrared Astronomical Satellite, NASA, Washington, DC

Renson P., 1978, A\&A 63, 125

Scargle D.J., 1982, ApJ 263, 835

Swingler D.N., 1989, AJ 97, 280

Terzan A., Bijaoui A., Ju K.H., Ounnas C. 1982, A\&AS 49, 715

Terzan A., Gosset E., 1991, A\&AS 90, 451

Vardya M.S., De Jong T., Willems F.J., 1986, ApJ 304, L32

Weinberg D.J., 1992, ApJ 384, 81

Whitelock P.A., Feast M.W., Catchpole R., 1991, MNRAS 248, 276 

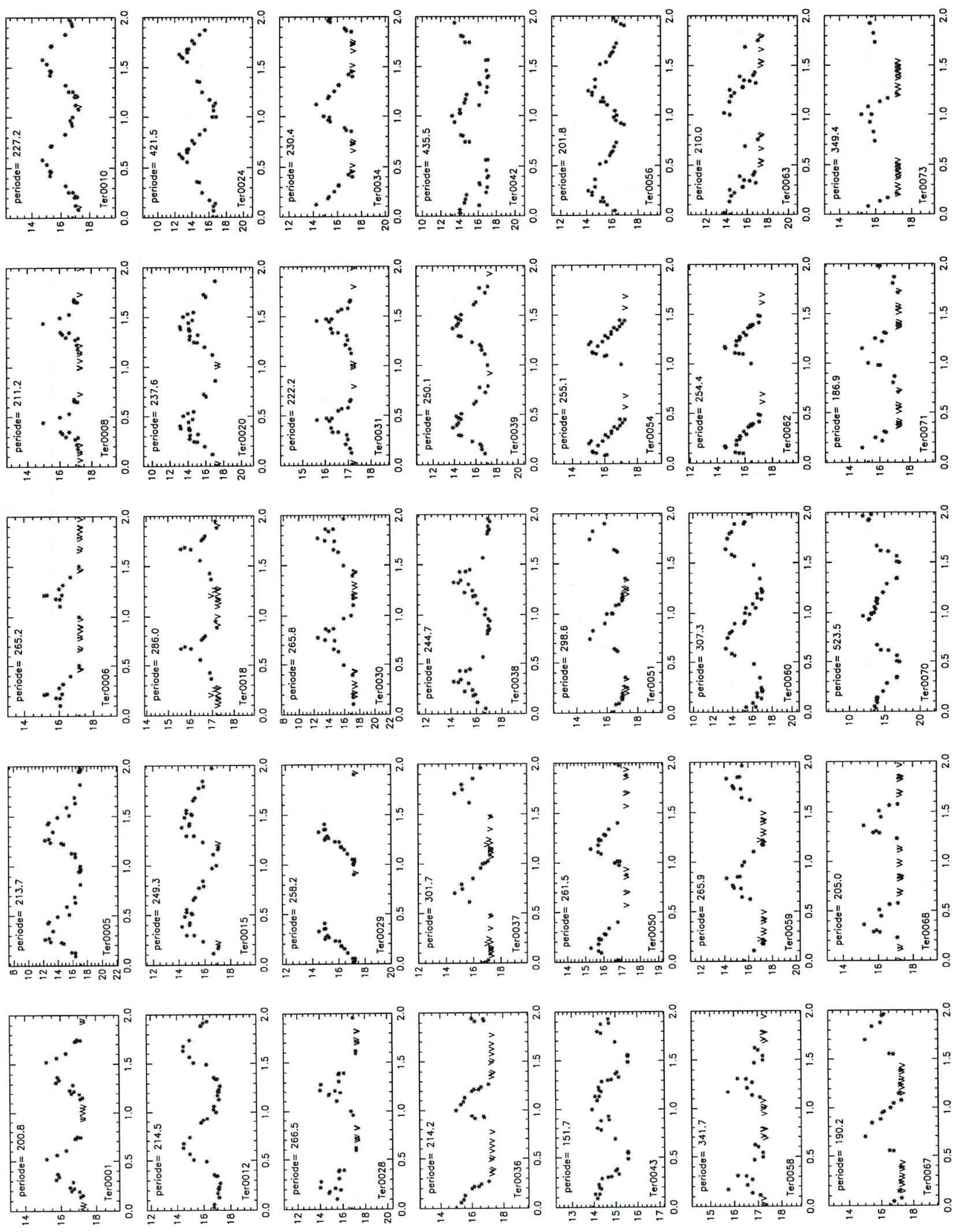

Fig. 5. Light curves of the Miras (only in electronic form) 

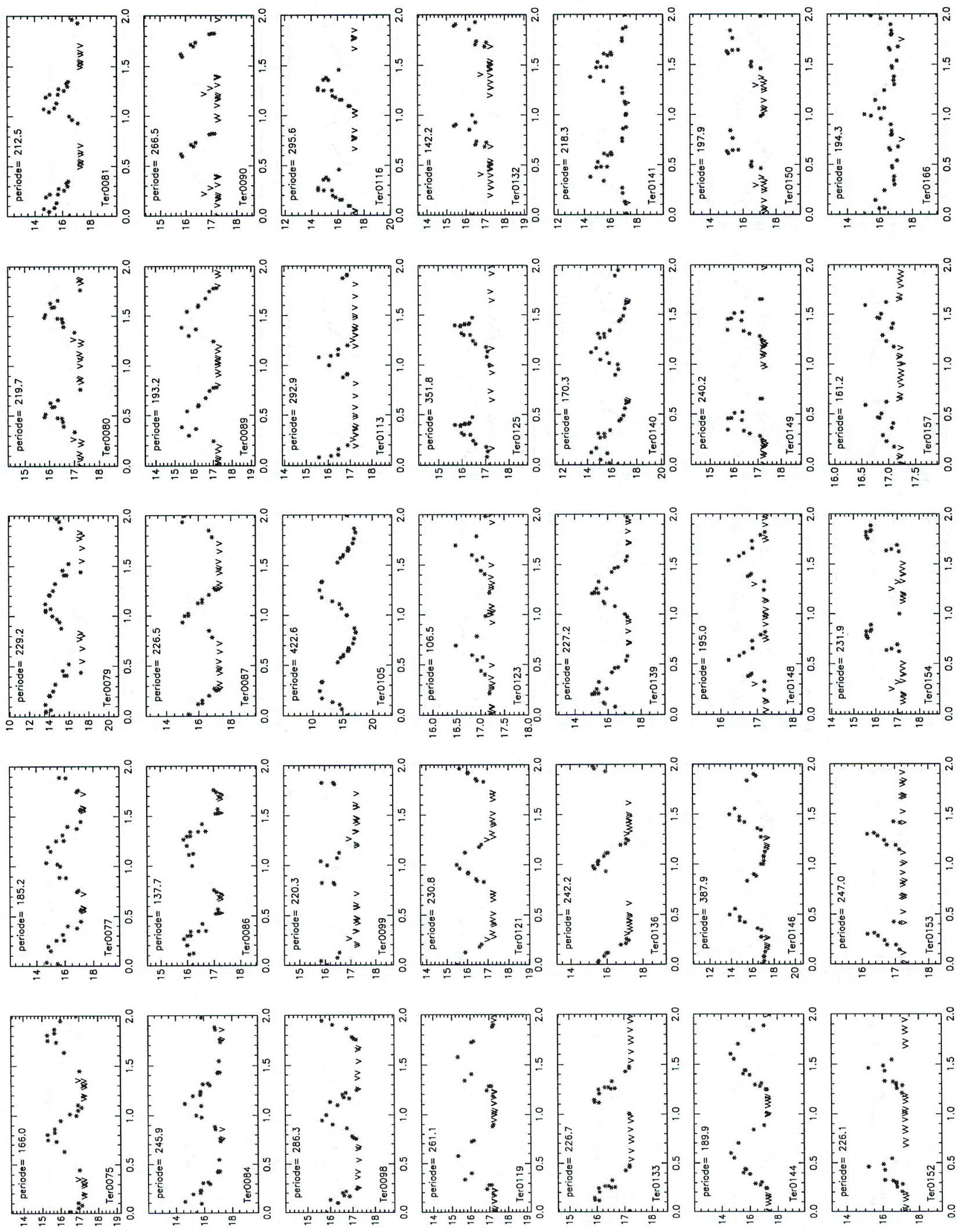

Fig. 6. continued (only in electronic form) 

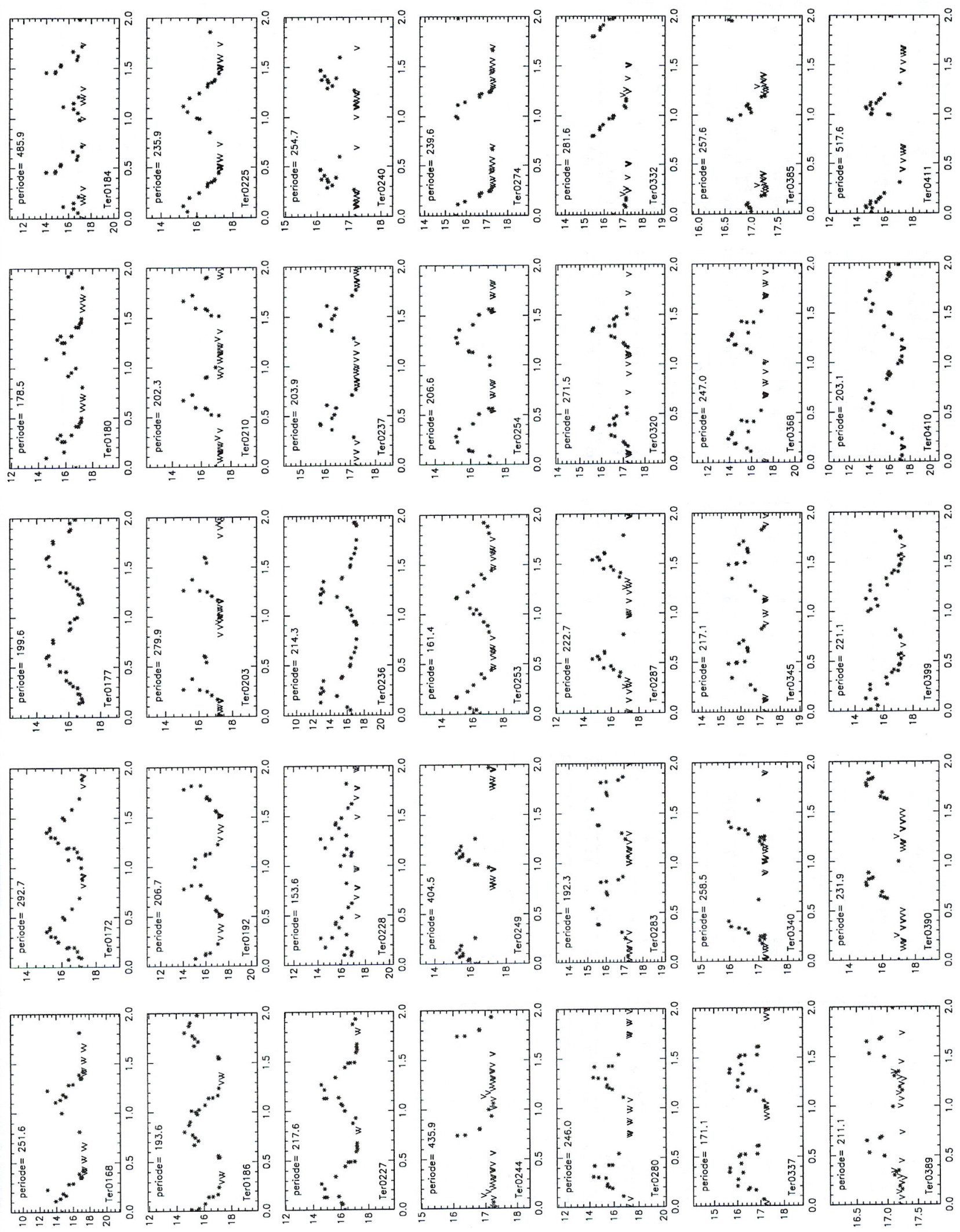

Fig. 7. continued (only in electronic form) 

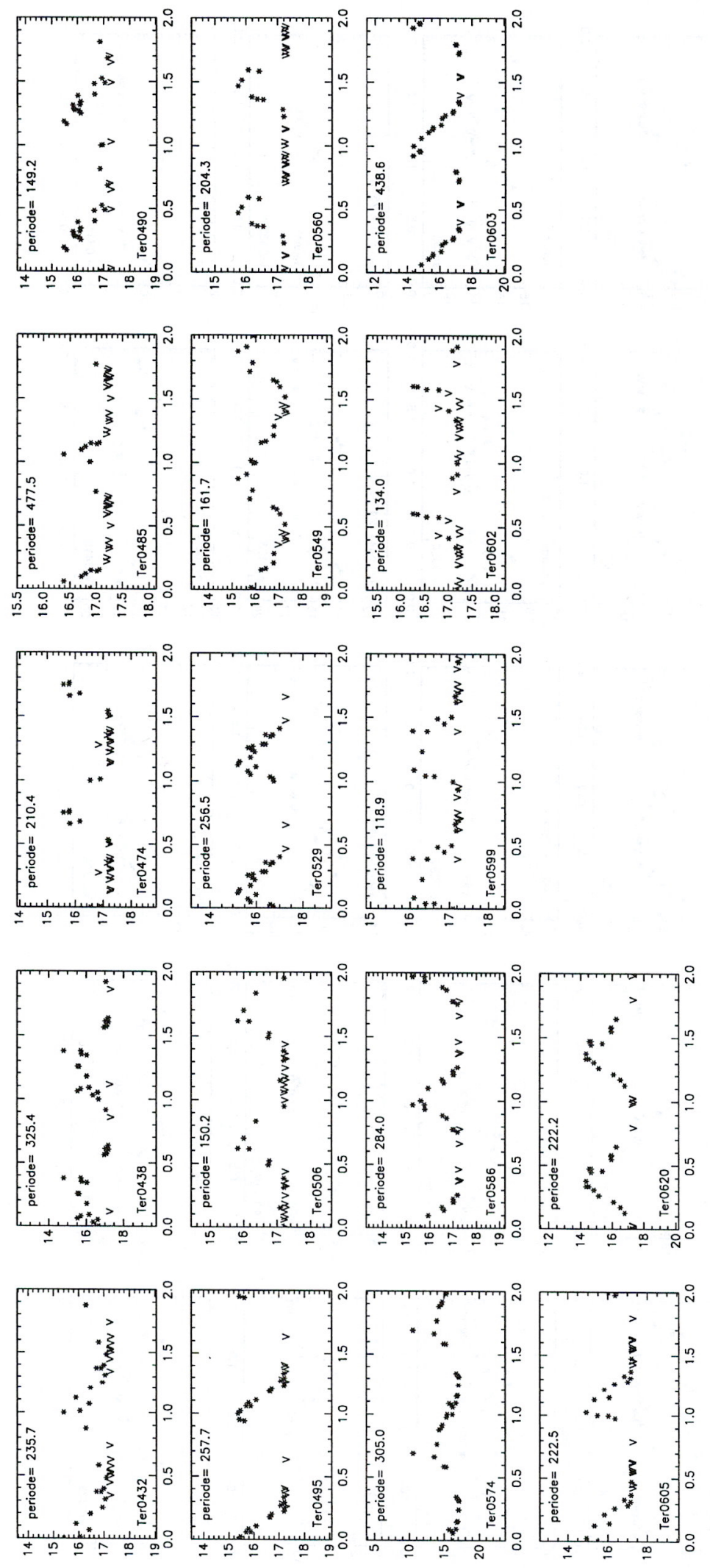

Fig. 8. continued (only in electronic form) 